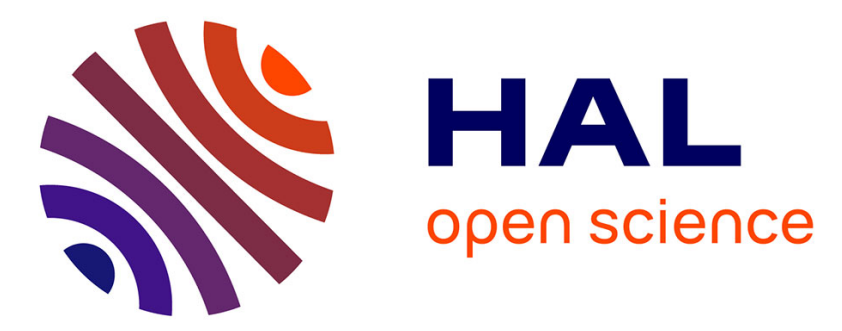

\title{
Symmetric Binary Random Signals With Given Spectral Properties
}

Bernard Picinbono

\section{To cite this version:}

Bernard Picinbono. Symmetric Binary Random Signals With Given Spectral Properties. IEEE Transactions on Signal Processing, 2016, 64 (19), pp.4952 - 4959. 10.1109/TSP.2016.2580537 . hal01376920

\section{HAL Id: hal-01376920 \\ https://hal-centralesupelec.archives-ouvertes.fr/hal-01376920}

Submitted on 5 Oct 2016

HAL is a multi-disciplinary open access archive for the deposit and dissemination of scientific research documents, whether they are published or not. The documents may come from teaching and research institutions in France or abroad, or from public or private research centers.
L'archive ouverte pluridisciplinaire HAL, est destinée au dépôt et à la diffusion de documents scientifiques de niveau recherche, publiés ou non, émanant des établissements d'enseignement et de recherche français ou étrangers, des laboratoires publics ou privés. 


\title{
Symmetric Binary Random Signals with Given Spectral Properties
}

Bernard Picinbono, Life Fellow, IEEE,

The pdf version of the final printed text can be obtained on request to the author at bernard.picinbono@1ss.supelec.fr

\begin{abstract}
The usual known methods for generating signals with given marginal probability and spectral properties cannot be applied to binary signals widely used in theoretical problems and communications systems. To overcome this difficulty we first present some structural properties of power spectral densities, enabling the precise definition of the concept of spectral properties. This allows us to introduce a new method valid for symmetric binary random signals. This method uses some specific properties of filters with random impulse responses. Results of computer simulations show clearly the good performance of this method. Some extensions by using random thinning can further improve its performance.
\end{abstract}

\section{Index Terms}

Probability distribution, random coefficients, Bernoulli signals, non Gaussian signals and noise, signal and noise modeling, statistical signal analysis.

\section{INTRODUCTION}

Binary signals are continuous or discrete time signals taking only two possible values. They play a very important role in communication theory and are widely used in practical digital systems. Sequences of values 0 or 1 are the best examples of discrete time (DT) binary signals. They are commonly used in

Manuscript received January, 2016.

B. P. is with the Laboratoire des Signaux et Systèmes (L2S), a joint laboratory of the C.N.R.S. and the École Supérieure d'Électricité, Plateau de Moulon, 3 rue Joliot-Curie 91192, Gif sur Yvette, France. The L2S is associated with the University of Paris-Sud, France. E-mail: bernard.picinbono@1ss.supelec.fr, . 
Information Theory where the basic model of communication channel is the binary symmetric channel and the input and output of which are binary signals. The same situation arises in computer science and communication technology. In particular number of studies are devoted to channel equalization where the analyzed signals are binary. This is also the case in various problems of detection and estimation especially in radar or sonar systems. In this context the advantage of binary signals is the absence of amplitude variation which introduces a better control of reverberation or clutter effects. Finally in passive sonar the source detection with coincidence polarity correlation systems makes use of the covariance function $(\mathrm{CF})$ of binary signals, This is the origin of number of studies on the calculation of the $\mathrm{CF}$ of clipped signals which are binary and symmetric. This justifies the interest of addressing the problem of generating binary signals with given spectral properties.

In what follows, we shall only consider symmetric binary random signals characterized by the facts that they take only the two values $\pm a$, where $a$ is a real amplitude, and that they are zero mean-valued. This implies that they take the values $\pm a$ with the same probability $1 / 2$, which in turns means that their marginal probability distribution is a symmetric Bernoulli distribution. Note that the square of such signals is constant and equal to $a^{2}$, which then is also the value of the variance of these signals. In the stationary case, which is assumed in all what follows except when it is otherwise indicated, the second order properties of such signals are characterized by their covariance function $\gamma(\tau)$ in the continuous time (CT) or $\gamma_{k}$ in the discrete time (DT) case.

Let us present some additional comments about our statistical assumptions. The stationary assumption is necessary as soon as we talk about spectral properties deduced from the CF. Indeed its definition requires at least second-order stationarity. For the discussion that follows it is also important to assume that the analyzed signals enjoy some ergodic properties. In short this means that ensemble averages which are deduced from the statistical distribution of signals are equal to time averages which are obtained in experimental or simulated measurements. We shall see below that all the signals introduced in our analysis are more or less directly deduced from sequences of independent and identically distributed (IID) random variables (RV). It is well known that such sequences satisfy the so called "strong mixing condition" ensuring the validity of the strong law of great numbers, in such a way that they are weakly and strongly ergodic. It is possible to verify that the signals presented in this paper are ergodic, but the proof is technically rather complicated and does not belong to the main ideas presented below. Ergodicity is here considered as a valid assumption. As a consequence of these statistical assumptions some signals are excluded from the scope of our analysis. It is especially the case of periodic signals widely used in many applications. Indeed they cannot be stationary because they are entirely determined as soon as they are known on a finite time interval defining their period. In the DT case this means that such signals are 
completely described as vectors of finite dimension. Furthermore periodic signals have a line spectrum, which excludes the existence of a power spectral density which is the main subject of what follows.

It is clear that a CT symmetric binary random signal (SBRS) is defined by its value at an arbitrary time instant (origin of time) and by the sequence of points $t_{i}$ at which its sign changes. This set of times constitute a random point process, and then to any point process it is possible to associate a corresponding SBRS. When this process is a stationary Poisson process of density $\mu$, the corresponding SBRS is the random telegraph signal widely used in the literature (see p. 334 of [1]). It is a CT random signal with the exponential CF $\gamma(\tau)=a^{2} \exp (-2 \mu|\tau|)$. It is, however, rather complicated to deduce the CF of a CT SBRS from the statistical properties of the point process used for its construction. We then do not use this approach in the following.

The problem addressed in this paper is the following: is it possible to construct a SBRS with given covariance or given spectral properties, as specified below? This is a particular case of the known problem of constructing a random signal with arbitrary marginal probability distribution and covariance function. In Section II we shall present the most classical ideas leading to the possible solutions of this problem and show that they cannot always be applied to SBRS either because they introduce assumptions that are not satisfied by these signals or because they give solutions that do not satisfy some basic properties that must be satisfied by any random signal. In Section III we motivate the concept of spectral properties referred to in the title of this paper. We show in particular that it is possible to introduce in the set of power spectral densities of unit power signals an equivalence relation which associates to any power spectral density (PSD), sometimes simply called spectrum, a set of other spectra considered as spectrally equivalent. This yields a partition of the set of PSDs into spectral equivalence classes and we show that this equivalence relation can be expressed in terms of the structures of the signals. By using the random filtering partially introduced in [2] we show in Section IV that for a given $\operatorname{PSD} \Gamma(\nu)$ and its spectral equivalence class introduced in Section III, it is always possible to construct at least one SBRS with a PSD belonging to this equivalence class, that is, with the given spectral properties. We extend this result under some conditions showing that it is always possible to construct a SBRS with a given PSD. Results of some simulations and computer experiments are in very good agreement with the theoretical analysis. Finally, we extend these results in the particular case of signals that are simultaneously symmetric binary and moving average of order 1, usually noted MA(1), and this extension is illustrated by some computer simulations. 


\section{Statement AND History of the Problem}

The most fundamental statistical properties describing a zero-mean stationary signal $X(t)$ are its marginal probability distribution and its CF. The first is the distribution of the random variable (RV) $X(t)$. For a stationary signal, this distribution is independent of time. Note immediately that these two properties are coupled, in such a way that they cannot in general be defined independently. For example both the $\mathrm{CF}$ and the marginal probability distribution determine the value of the variance of the signal.

The problem of generating a random signal with given marginal distribution and CF was first addressed in [3], [4], [5] long time ago. The main idea was to process a Gaussian white noise by a sequence of a linear filtering and an instantaneous nonlinearity. The covariance properties are specified by the input linear filter while the marginal probability distribution determines the structure of the nonlinearity. It is clear, however, that the two operations are in general coupled because the nonlinearity transforms the $\mathrm{CF}$, which implies that the structure of the linear filter cannot in general be determined without taking into account the structure of the nonlinearity. As a consequence the method can fail because it can lead to introduce CFs that are not non-negative definite (NND), a necessary property of such functions.

This especially appears in the case discussed in this paper where the marginal distribution of the signal is a Bernoulli distribution. Indeed in order to transform a Gaussian signal into a SBRS the nonlinearity which clearly seems appropriate is the perfect clipping. It transforms a signal $X(t)$ into another one $Y(t)$ equal to 1 if $X(t)>0$, to 0 if $X(t)=0$, and to -1 otherwise. If $X(t)$ is Gaussian and zero mean valued this nonlinearity preserves the zero mean value and the output $\mathrm{CF}$ is given by the famous expression $\gamma_{Y}(\tau)=(2 / \pi) \arcsin \left[\rho_{X}(\tau)\right]$, where $\rho_{X}(\tau)$ is the normalized CF of $X(t)$ (see p. 141 of [1]). By inverting this expression we get $\rho_{X}(\tau)=\sin \left[(\pi / 2) \gamma_{Y}(\tau)\right]$. But for a given NND function $\gamma_{Y}(\tau)$ there is no reason for $\rho_{X}(\tau)=\sin \left[(\pi / 2) \gamma_{Y}(\tau)\right]$ to be NND, which makes impossible to generate the corresponding signal $X(t)$. An example of this situation is discussed in the last section.

A completely different solution of this problem was recently proposed in [6]. Its main advantage is of decoupling the effects of the two constraints related to the probability distribution and the covariance properties. Its major inconvenience however is the fact that this method requires that the marginal probability density function (PDF) of the signal be symmetric and infinitely divisible. The first assumption is obviously satisfied by symmetric SBRS but, on the other hand, that is not at all the case for the latter assumption. This makes it impossible to use this method in the problem addressed in this paper. Finally another approach for this problem was recently presented in the case of autoregressive and moving average (ARMA) signals [2]. By using filters with random coefficients it is possible, under very general conditions, to generate ARMA signals, i. e. signals with power spectrum having the structure of a rational 
fraction, with arbitrary probability distribution. It is this approach that will be extended without the ARMA constraint and for SRBS.

According to the two more recent papers on this kind of problem it appears that there are not a great number of references in the signal processing literature related to this subject. There are, however, many references on similar problems in the statistical literature where Bernoulli distribution is of great importance. The most significant references in our opinion are [7], [8] and the references therein. But an important difference appears immediately. These papers are mainly devoted to the study of vectors of finite dimension which is the standard situation in statistical problems, while spectral properties analyzed in signal theory require the use of stationary signals or vectors of infinite dimension. This implies that the use of linear or nonlinear filtering especially common in signal proceeding literature is rarely used in pure statistical literature. This explains why most of the papers of this origin are not of a real interest in the problem addressed in this paper.

\section{Concept of Spectral Properties}

\section{A. Definition of Minimum DSP}

A DT random signal $X_{k}$ is said to be of unit power (UP) if its variance $\sigma^{2}$ satisfies $\sigma^{2}=1$. This is clearly the case of a SBRS taking only the values \pm 1 . In this section we consider only UP signals.

This implies that their corresponding PSDs, or spectra, $\Gamma(\nu)$ are normalized, that is, $\int_{-1 / 2}^{1 / 2} \Gamma(\nu) d \nu=1$. Furthermore we assume in all what follows that the PSDs $\Gamma(\nu)$ used are bounded and continuous in the interval $[-1 / 2,1 / 2]$. This obviously excludes the case of PSDs containing spectral lines or random signals (RS) with periodic components. Let $\mu$ be the minimum of the PSD $\Gamma(\nu)$ in the interval $[-1 / 2,1 / 2]$. It is clear that $\mu \geq 0$ because any PSD is a nonnegative function of $\nu$. Furthermore $\mu \leq 1$ because $\Gamma(\nu)$ is normalized as PSD of an UP signal. Thus, $0 \leq \mu \leq 1$. If $\mu=0$ we say that $\Gamma(\nu)$ is a minimum PSD. If $\mu=1$ the PSD is equal to 1 and the corresponding signal is white. Let us introduce the PSD $\check{\Gamma}(\nu)$ defined by

$$
\check{\Gamma}(\nu) \triangleq \frac{1}{1-\mu}[\Gamma(\nu)-\mu] .
$$

It is clear that this function is a normalized and minimum PSD. It is called the minimum PSD associated with $\Gamma(\nu)$. Conversely we have

$$
\Gamma(\nu)=(1-\mu) \check{\Gamma}(\nu)+\mu
$$

\section{B. Equivalence Class of Spectra}

Let $\check{\Gamma}(\nu)$ be a given minimum PSD and $\Gamma_{\mu}(\nu)$ the spectrum defined from $\check{\Gamma}(\nu)$ by $(2)$ where $\mu \in[0,1]$. To the set of possible values of $\mu$ we can associate a set of spectra having the same minimum PSD. 
This set of spectra is clearly an equivalence class where the equivalence relation between two PSDs means that they have the same minimum PSD $\check{\Gamma}(\nu)$. This equivalence class is denoted by $\mathcal{C}_{\check{\Gamma}}$ and called an equivalence spectral class. The set of all these equivalence classes defines a partition of the set of normalized PSDs.

It is clear that all these relations and concepts can be expressed in terms of the CF $\gamma_{k}$, the inverse Fourier transform of $\Gamma(\nu)$. In the time domain the relation (2) becomes

$$
\gamma_{k}=(1-\mu) \check{\gamma}_{k}+\mu \delta[k]
$$

where $\delta[k]$ is the Kronecker delta symbol taking the value 1 for $k=0$ and 0 otherwise. This exhibits two of the main properties of the CFs $\gamma_{\mu}[k]$ associated with the spectra belonging to the equivalence class $\mathcal{C}_{\check{\Gamma}}$ : all these CFs satisfy $\gamma_{\mu}[0]=1$, which means that they are CFs of UP signals and for $k \neq 0$ they are proportional. This will play an important role in the following analysis.

\section{Spectral Properties of a Signal}

By definition, all the elements of an equivalence class are equivalent and since this class is defined by a specific minimum normalized PSD $\check{\Gamma}(\nu)$, we shall say that all these elements have the same spectral properties. In other words finding a signal with specific spectral properties defined by $\check{\Gamma}(\nu)$ is equivalent of finding an element of the equivalence class $\mathcal{C}_{\check{\Gamma}}$.

It is also interesting to write these relations in terms of the signals themselves. Let $X_{k}$ be a UP SBRS taking only the values \pm 1 such that its PSD $\Gamma_{X}(\nu)$ belongs to $\mathcal{C}_{\check{\Gamma}}$. This means that (2) holds, where $\Gamma$ is replaced by $\Gamma_{X}$. Let now $Y_{k}$ be defined by

$$
Y_{k}=B_{k} X_{k}+\bar{B}_{k} W_{k}
$$

where $B_{k}$ is a sequence of IID Bernoulli RVs taking only the values 1 and 0 with the probabilities $p$ and $q=1-p$ respectively and $\bar{B}_{k}=1-B_{k}$, and $W_{k}$ is a white SBRS taking the values \pm 1 independent of $B_{k}$. We further assume that $B_{k}$ and $W_{k}$ are independent of $X_{k}$. It results from these assumptions that $Y_{k}$ also is a SBRS taking the values \pm 1 and that for $i \neq 0$ the CFs of $Y_{k}$ and $X_{k}$ are related by $\gamma_{Y}[i]=p^{2} \gamma_{X}[i]$. On the other hand the fact that $Y_{k}$ is UP implies $\gamma_{Y}[0]=1$. This yields $\gamma_{Y}[k]=p^{2} \gamma_{X}[k]+\left(1-p^{2}\right) \delta[k]$, where $\delta[k]$ is the Kronecker delta symbol taking the value 1 for $k=0$ and 0 otherwise. After a Fourier transformation we obtain

$$
\Gamma_{Y}(\nu)=p^{2} \Gamma_{X}(\nu)+1-p^{2}
$$

Since $\Gamma_{X}(\nu)$ belongs to $\mathcal{C}_{\breve{\Gamma}}$ it satisfies (2) where $\mu$ is replaced by $\mu_{X}$, and using (5) yields

$$
\Gamma_{Y}(\nu)=p^{2}\left(1-\mu_{X}\right) \check{\Gamma}(\nu)+1-p^{2}\left(1-\mu_{X}\right),
$$


which shows that $\Gamma_{Y}(\nu)$ also belongs to $\mathcal{C}_{\check{\Gamma}}$. Let $\mu_{X}$ and $\mu_{Y}$ be the minimum values of $\Gamma_{X}$ and $\Gamma_{Y}$ respectively. We deduce either from (5) or (6) that

$$
\mu_{Y}=1-p^{2}\left(1-\mu_{X}\right)
$$

which shows that as $p$ increases from 0 to $1, \mu_{Y}$ decreases from 1 to $\mu_{X}$. As a result $\mu_{Y}>\mu_{X}$, an inequality useful in the following discussion.

If the PSD $\Gamma_{X}(\nu)$ is minimum, which is specified by $\mu_{X}=0$, or, according to (2), if $\Gamma_{X}=\check{\Gamma}$, it is then possible to use (5) to reach all the elements of the equivalence class $\mathcal{C}_{\check{\Gamma}}$ by using (6) with $\mu_{X}=0$ and the appropriate value $p=\sqrt{1-\mu_{Y}}$ deduced from (7). This value used in (4) yields the corresponding signal $Y_{k}$. This shows that to each element of $\mathcal{C}_{\check{\Gamma}}$ we can associate a SBRS having this element as PSD, which was the initial purpose of this paper.

On the other hand if $\Gamma_{X}(\nu)$ is not minimum, which means that $\mu_{X}>0$, it is only possible with (6) to reach a subset of $\mathcal{C}_{\check{\Gamma}}$. It is the set of PSDs $\Gamma_{Y}(\nu)$ given by (6) or PSDs of $\mathcal{C}_{\check{\Gamma}}$ such that their minimum $\mu_{Y}$ is given by (7). Their only constraint is that $\mu_{Y}>\mu_{X}$ and for any value $\mu_{Y}$ satisfying this inequality there exists a signal $Y_{k}$ deduced from $X_{k}$ by (4) such that the appropriate value of $p$ is given by (7) that is, $p=\sqrt{\left(1-\mu_{Y}\right) /\left(1-\mu_{X}\right)}$.

The structure of (4) leads us to introduce in our discussion the concept of random filtering.

\section{Binary Signals with Given Spectral Properties by Random Filtering}

There is a case where the problem addressed in this paper has an evident and simple solution by using linear filtering. This is in the case of Gaussian signals for which we can independently treat the problem of the covariance generation and that of the probability distribution. This comes from the well-known fact that a Gaussian signal remains Gaussian when it is processed by a linear filter and since all its probability distribution is completely determined by its covariance it suffices to process a white Gaussian noise with a linear filter to obtain a Gaussian signal, the second order properties of which being entirely determined by the structure of this filter. Let us first briefly recall this classical procedure.

Consider a causal linear filter defined by its impulse response (IR) $h_{k}, k \geq 0$, or its frequency response $H(\nu)$, the Fourier transform of $h_{k}$. For the following discussion we introduce two parameters of this filter defined by

$$
S_{1} \triangleq \sum_{k=0}^{\infty}\left|h_{k}\right| \quad ; \quad S_{2} \triangleq \sum_{k=0}^{\infty} h_{k}^{2}
$$


We assume that $S_{1}<\infty$ which is the necessary and sufficient condition for the filter to be stable in the BIBO sense. Suppose now that the input is a zero mean Gaussian white noise $W_{k}$ of unit power. The corresponding output $\bar{X}_{k}$ is given by

$$
\bar{X}_{k}=\sum_{l=0}^{\infty} h_{l} W_{k-l} .
$$

This is a zero-mean Gaussian signal with statistical properties entirely defined by its CF given by

$$
\gamma_{\bar{X}}[g]=\sum_{k=0}^{\infty} \sum_{l=0}^{\infty} h_{k} h_{l} \delta[g-k+l],
$$

where $g$, last letter of lag, is an integer and $\delta[k]$ is the Kronecker delta signal defined before. In particular the output power is given by

$$
\sigma_{\bar{X}}^{2}=\gamma_{\bar{X}}[0]=\sum_{k=0}^{\infty} h_{k}^{2}=S_{2},
$$

and assuming that $S_{2}$ in (8) is 1 yields that the output signal is an UP Gaussian signal.

Suppose now that $W_{k}$ of (9) is a white UP SBRS. All the previous equations concerning the second order properties remain the same. On the other hand there is no reason for the output $\bar{X}_{k}$ to remain an UP SBRS, because the sum of binary random variables is no longer binary. It is then necessary to introduce another approach and the use of random filtering seems an interesting method. Random filtering appears when the IR of the filter becomes random. Random filters are used in various fields of Signal Processing [9], [10], [11]. The results presented in these papers, however, have no direct relationship with those used for our specific problem.

The principle of filtering, however, is so important in the problems of signal processing that we shall introduce a filtering procedure that preserves the probability distribution of the input while the transformation of the $\mathrm{CF}$ is very similar to the one appearing in the standard linear filtering and given by (10).

For this, consider the particular random IR defined as follows. Let $B_{k}$ be a strictly white noise, that is, a sequence of IID random variables. We assume furthermore that they are uniformly distributed in the interval $\left[0, S_{1}\right]$, where $S_{1}$ appears in (8). Consider also a partition of this interval in non-overlapping intervals $\Delta_{l}$ of measures $\delta_{l}=\left|h_{l}\right|$. The partition property is ensured because of (8). Let $c_{l}(x)$ be the indicator function of the interval $\Delta_{l}$, which means that $c_{l}(x)=1$, if $x \in \Delta_{l}$ and 0 otherwise. Finally let $\epsilon_{l}=\operatorname{sign}\left(h_{l}\right)$, or $\epsilon_{l}=1$ if $h_{l}>0$ and -1 otherwise. The input-output relationship defining the random filter used in this paper is

$$
X_{k}=\sum_{l=0}^{\infty} g_{l k} W_{k-l},
$$

where

$$
g_{l k}=\epsilon_{l} c_{l}\left(B_{k}\right) .
$$


We assume also that the signals $B_{k}$ and $W_{k}$ are independent. Since the functions $c_{i}(x)$ are indicator functions of subsets of a partition, they satisfy

$$
c_{i}(x) c_{j}(x)=\delta[i-j] c_{i}(x)
$$

where $\delta$ is the Kronecker delta signal defined before. It results from this relation that the sum (or the series) appearing in (12) contains in reality only one non-zero term. Indeed for each outcome $b_{k}$ of the random variable $B_{k}$ all the $g_{l k}$ are zero except the one for which $c_{l_{k}}\left(b_{k}\right)=1$. But the probability that $c_{i}\left(B_{k}\right)=1$ is the probability that $B_{k}$ belongs to the subset of $\left[0, S_{1}\right]$ defined by $c_{i}(x)=1$. Since $B_{k}$ is uniformly distributed in $\left[0, S_{1}\right]$, this probability is $\pi_{i}=\left|h_{i}\right| / S_{1}$. As a result $X_{k}$ defined by (12) takes the values $\epsilon_{i} W_{k-i}$ with the probability $\pi_{i}$. This is the fundamental property of the filtering defined by (12).

Its first direct consequence is that, since the input $W_{k}$ is binary symmetric, the output $X_{k}$ enjoys the same property. This follows directly from the previous remark that the only possible values of $X_{k}$ are $\epsilon_{i} W_{k-i}$ and $\epsilon_{i}$ also takes only the values \pm 1 . The symmetry comes from the fact that the expected value of $X_{k}$ is 0 , as it appears directly in (12).

Note, however, that this result does not mean that the input $W_{k}$ and the output $X_{k}$ have the same complete probability distributions. Indeed nothing is said about the joint probability distributions of the values of the signal at different times. In reality we shall see below that the CFs of these signals are not the same, which proves that the bivariate joint probability distributions are different.

The second direct consequence of (12) is that the CFs $\gamma_{\bar{X}}[g]$ and $\gamma_{X}[g]$ of $\bar{X}_{k}$ and $X_{k}$ respectively are proportional for $g \neq 0$.

The CF $\gamma_{\bar{X}}[g]$ of $\bar{X}_{k}$ is given by (10). Let us now calculate the CF $\gamma_{X}[g]$ of $X_{k}$ for $g \neq 0$. Since, as seen above, $m_{X}=0$, we have $\gamma_{X}[g]=\mathrm{E}\left[X_{k} X_{k-g}\right]$ and according to (12) we obtain

$$
\gamma_{X}[g]=\sum_{l_{1}=0}^{\infty} \sum_{l_{2}=0}^{\infty} \epsilon_{l_{1}} \epsilon_{l_{2}} \mathrm{E}\left[c_{l_{1}}\left(B_{k}\right) c_{l_{2}}\left(B_{k-g}\right)\right] \delta\left[g-l_{1}+l_{2}\right],
$$

where the expectation is taken with respect to $B_{k}$ and $B_{k-g}$ which are independent. It results from the definition of $c_{k}$ that $\mathrm{E}\left[c_{l}\left(B_{k}\right)\right]=\left|h_{l}\right| / S_{1}$, in such a way that

$$
\gamma_{X}[g]=\left(1 / S_{1}^{2}\right) \sum_{k=0}^{\infty} \sum_{l=0}^{\infty} h_{k} h_{l} \delta[g-k+l],
$$

which, according to (10), yields $\gamma_{X}[g]=\left(1 / S_{1}^{2}\right) \gamma_{\bar{X}}[g]$ for $g \neq 0$.

We now shall show that the spectra of $X_{k}$ and $\bar{X}_{k}$ belong to the same spectral equivalence class. To that end, note that for $g=0$ we have $\gamma_{X}[0]=\gamma_{\bar{X}}[0]=1$. For $X_{k}$ this comes from the fact that it is by construction a UP signal as indicated just above. For $\bar{X}_{k}$ this results from (11) and the assumption $S_{2}=1$. This can be summarized in a single expression which is 


$$
\forall g, \gamma_{X}[g]=\left(1 / S_{1}^{2}\right) \gamma_{\bar{X}}[g]+c \delta[g]
$$

where $c=1-\left(1 / S_{1}^{2}\right) \gamma_{\bar{X}}[0]$ and $\delta[k]$ is the Kronecker delta symbol. Since $\gamma_{\bar{X}}[0]=1$, as noted just above, we get $c=1-1 / S_{1}^{2}$. Let us now show that $S_{1}^{2}>1$. For this we start from (11) and we transform this expression in terms of the frequency response $H(\nu)$ of the filter defined by $h_{k}$. This yields

$$
S_{2}=\int_{-1 / 2}^{1 / 2}|H(\nu)|^{2} d \nu=1 .
$$

Since $H(\nu)=\sum h_{k} \exp (-j \omega k), \omega=2 \pi \nu$, we deduce from (8) that $|H(\nu)|<S_{1}$, which yields $S_{1}^{2}>$ $S_{2}=1$. Taking the Fourier transform of (17) yields the same relation in terms of PSDs, that is

$$
\Gamma_{X}(\nu)=\alpha \Gamma_{\bar{X}}(\nu)+1-\alpha
$$

where $\alpha=1 / S_{1}^{2}$ and $0<\alpha<1$.

Comparing with (2), (5), or (6) we can say that the PSDs of $X_{k}$ and $\bar{X}_{k}$ belong to the same spectral equivalence class, as defined in the previous section. Consequently we can say that $X_{k}$ and $\bar{X}_{k}$ have the same spectral properties.

Let us review the steps of the procedure. Our purpose is to construct a UP SBRS with given spectral properties. We start from a given PSD $\Gamma(\nu)$. Factorizing this function in the form $|H(\nu)|^{2}$ defines a linear filter. Its impulse response $h_{k}$ can be deduced from $H(\nu)$. It is well known that this solution is not unique because of the degrees of freedom concerning the phase of $H(\nu)$, which plays no role in the structure of $\Gamma(\nu)$. However, there is a standard procedure used in prediction problems in which the factorization problem has a unique solution when one introduces the condition that $H(\nu)$ is a filter that is causal and such that its inverse is causal. The corresponding result is called the strong factorization of $\Gamma(\nu)$ (see p. 222 of [1]). This form of factorization is not necessary in our problem. Its interest, however, is that, because of its importance in prediction and estimation problems (Wiener filtering), there are a great number of methods and algorithms for its realization. Having calculated the impulse response $h_{k}$ we can construct the random filter used in (12). Its output is a unit power symmetric binary random signal and according to (19) its PSD belongs to the same spectral equivalence class as $\Gamma(\nu)$. As a consequence the $\mathrm{RS} X_{k}$ is a solution of the problem addressed at the beginning of this paper: it is binary symmetric and has the desired spectral properties.

At this step of the reasoning we can address a more restrictive question : Is it possible to construct an UP SBRS such that its PSD is not only in the equivalence class $\mathcal{C}_{\Gamma}$ defined by $\Gamma(\nu)$ but is also equal to $\Gamma(\nu)$ ? This is in fact the question addressed at the end of the previous section and we shall use more precisely the results presented here. 
For this, let $\check{\Gamma}(\nu)$ be the minimum PSD of $\mathcal{C}_{\Gamma}$. The elements of this equivalence class are given by (2) rewritten here

$$
\Gamma_{\mu}(\nu)=(1-\mu) \check{\Gamma}(\nu)+\mu
$$

which shows that they depend only on the parameter $\mu, 0<\mu<1$. Then, our purpose is to find values of $\mu$ such that $\Gamma_{\mu}(\nu)$ is the PSD of an UP SBRS that can be constructed. For this purpose we begin by calculating the factorization of $\check{\Gamma}(\nu)$ yielding as previously the coefficients $h_{k}$ used to define the random filter generating the signal $X_{k}$ by (12). The PSD of this signal is given by (19) taking here the form

$$
\Gamma_{X}(\nu)=\alpha \check{\Gamma}(\nu)+1-\alpha
$$

with $\alpha=1 / S_{1}^{2}$. From this signal we calculate $Y_{k}$ defined by (4). Its PSD is given by (5) and is defined by the parameter $p$ appearing in the definition of this equation. Since the minimum value of $\check{\Gamma}(\nu)$ is zero we deduce from (21) that $\mu_{X}=1-\alpha$. Applying (7) yields

$$
\mu_{Y}=1-\alpha p^{2}
$$

which implies that $1-\alpha<\mu_{Y}<1$. We deduce that for any $\mu_{Y}$ satisfying these inequalities we can calculate a value of $p$ given by

$$
p=\sqrt{(1 / \alpha)\left(1-\mu_{Y}\right)} .
$$

This quantity is the probability of the Bernouli variable $B_{k}$ used in (4) for computing $Y_{k}$. This signal satisfies the initial requirements: it is UPBS and its PSD is $\Gamma_{Y}(\nu)$ defined by

$$
\Gamma_{Y}(\nu)=\left(1-\mu_{Y}\right) \check{\Gamma}(\nu)+\mu_{Y}
$$

where $\check{\Gamma}(\nu)$ and $\mu_{Y}, 1-\alpha<\mu_{Y}<1$, are given in advance.

On the other hand it seems that there is no simple procedure to solve the same problem for the PSDs of $\mathcal{C}_{\Gamma}$ defined by (24) when $\mu_{Y}<1-\alpha$.

\section{Simulations AND EXPERIMENTS}

Let us first present the sequence of operations used in order to simulate the procedure of random filtering presented above. Let $\Gamma(\nu)$ be a given PSD. The purpose is to generate a UP binary random signal $X_{k}$ that is stationary and such that its PSD belongs to the equivalence class defined by $\Gamma(\nu)$. For this we use the following sequences of steps.

1. Factorization of the spectrum or search of the frequency response $H(\nu)$ of a causal linear filter such that $\Gamma(\nu)=|H(\nu)|^{2}$. This is a classical problem used for example in Wiener prediction where the 
filter must be not only causal but with minimum phase, condition not required in our problem. There are number of possible algorithms for this purpose.

2. Calculation of the parameters of the random filtering. It is defined by the function $g_{l k}$ given by (13). In this equation $\epsilon_{k}=\operatorname{sign}\left(h_{k}\right)$, and $B_{k}$ a strictly white noise with a marginal uniform distribution in $\left[0, S_{1}\right]$ deduced from (8). There are number of possible computer methods to generate sequence of values of such a signal.

3. The input of this system of random filtering is a signal $W_{k}$ which is a sequence of IID binary RVs taking the values \pm 1 . This sequence is assumed to be independent of $B_{k}$. There are then two input signals $B_{k}$ and $W_{k}$ and the output $X_{k}$ is given by (12). This calculation is clearly the most complicated part of the procedure. In the simulations presented below the calculation follows exactly the expression (12) which is an extension of the classical convolution. But, as for the convolution, it is possible to introduce fast algorithms not discussed since this work is more devoted to the principle of the method rather than to its refinements. The signal $X_{k}$ obtained with this method has the properties required at the beginning of the procedure.

4. According to some properties of the PSD $\Gamma(\nu)$, it is possible to answer the question addressed at the end of the previous section and to verify whether or not this method allows us to reach a signal $X_{k}$ whose the PSD is exactly $\Gamma(\nu)$.

We now present some experimental results on simulated data illustrating the previous theoretical analysis. Suppose that the input signal $W_{k}$ appearing in (9) and (12) is a strictly white SBRS, $i$. $e$. a sequence of IID random variables with zero mean value and taking only the values \pm 1 . Such a noise can easily be simulated on a computer and a segment of its trajectory appears in Fig. 1. This figure clearly shows that this signal is binary and takes only the values \pm 1 . A statistical analysis of a set of approximately $10^{7}$ successive values of this signal confirms some of its expected properties. It appears that its mean value is zero, which implies a symmetric probability distribution. Furthermore a second order analysis shows that the values of this signal are uncorrelated, which shows that the signal is, as expected, a white UP SBRS.

Consider now the minimum normalized PSD defined by

$$
\check{\Gamma}_{Y}(\nu)=\frac{1}{10} \frac{\sin ^{2}(10 \pi \nu)}{\cos ^{2}(\pi \nu)}
$$

It is easy to verify that its integral in the frequency domain $|\nu| \leq 1 / 2$ is 1 , which justifies the term normalized, and it is obvious that the minimum $\mu$ of this PSD is 0 , which justifies the term minimum. It is the PSD of the signal $\bar{X}_{k}$ given by (9) when the input is an unit power white signal and when the impulse response $h_{k}$ of the filter is $h_{k}=(1 / \sqrt{10})(-1)^{k}$ for $0 \leq k \leq 9$ and $h_{k}=0$ otherwise. The 
quantities $S_{1}$ and $S_{2}$ of (8) are equal to $\sqrt{10}$ and 1 respectively. This last value ensures that the output is a UP signal.

A trajectory of this signal $\bar{X}_{k}$ appears in Fig. 1 with the name $Y_{k}$ and exhibits the following main properties. This signal takes only discrete values and applying (9) to an input taking only the values \pm 1 shows that the output of this filter takes only the values $\pm(1 / \sqrt{10}) 2 k, 0 \leq k \leq 5$. This appears on the sequence $S Q$ of 10 successive values of this simulated signal having the form

$$
S Q=(1 / \sqrt{10})\left[\begin{array}{llllllllll}
4 & -6 & 6 & -8 & 10 & 6 & -4 & -2 & 0 & 2
\end{array}\right] .
$$

This clearly shows that the binary property of the input $W_{k}$ is not preserved after the classical linear filtering (convolution) defined by (9). Furthermore a statistical analysis of a long sequence of values of this signal shows that, as expected, its mean value is 0 and its variance is 1 . It belongs then to the class of UP signals introduced above.

It remains for us to consider the case of the signal $X_{k}$ defined by (12). A section of its trajectory appears in Fig. 1. As expected, this signal is binary and takes only the values \pm 1 . This obviously implies that its variance is 1 which means that, like $X_{k}$, it is of unit power. A statistical analysis of a long sequence shows that its mean value is 0 , which implies that it is symmetric, and thus is, like the input $W_{k}$, an UP SBRS. But their second-order statistical properties are different and we shall now verify that, as expected, $\bar{X}_{k}$ and $X_{k}$ have the same spectral properties.

For this purpose it suffices to verify that, according to (16), their covariances are proportional for $g \neq 0$. Results of covariance measurements are presented in Table 1.

In line 1 we present the values of the normalized theoretical covariance function (CF) given by (10) applied when $W_{k}$ is white and when the filter is the one used in experiments reported in Fig. 1. This CF is normalized because $\bar{X}_{k}$ is an UP signal. As previously noted, this comes from the property $S_{2}=1$ of the filter used in our experiments. These values appear for $0 \leq g \leq 12$, but $\gamma_{\bar{X}}[g]=0$ for $g \geq 10$. Lines 2 and 3 are experimental values obtained when the input white noise $W_{k}$ is a SBRS as in the experiments reported in Fig. 1. The results of lines 2 and 3 correspond to the signals $\bar{X}_{k}$ (filter (9) or convolution) and $X_{k}$ of (12) respectively.

Comparison between lines 1 and 2 shows that the results are exactly the same which means that the measured CF of the signal $\bar{X}_{k}$ corresponds exactly to its theoretical value given by (10). On the other hand the purpose or the results of line 3 is to verify that, according to (16), the CFs of $\bar{X}_{k}$ and of $X_{k}$ are proportional for $g \neq 0$. For the clarity, we note that (17) can be written $\gamma_{X}[g]=\alpha \gamma_{\bar{X}}[g]$ with $\alpha=1 / S_{1}^{2}$. It results from this that $(1 / \alpha) \gamma_{X}[g]$ is theoretically equal to $\gamma_{\bar{X}}[g]$ for $g \neq 0$ and line 3 presents the results of measurements of $(1 / \alpha) \gamma_{X}[g]$, which simplifies the comparison with lines 1 and 2 . We observe that 
the experimental results corresponds with a good precision to theoretical values. The small differences between them comes from statistical errors due to the structure of the random filter (12) introducing a randomness that was not present in the classical convolution. We have verified that these additional statistical errors decrease after increasing of the duration of the covariance measurements.

In concluding this section we can say that most of the results obtained by the theoretical analysis are in quite good agreement with those obtained by measurements on simulated data. This confirms that the method of random filtering introduced above is a quite a good way to generate signals with specified spectral properties.

TABLE 1. First TWELVE VAlues of

NORMALIZED COVARIANCE FUnCTIONS

\begin{tabular}{cccccccc}
\hline$\gamma[g] \backslash g$ & 0 & 1 & 2 & 3 & 4 & 5 & 6 \\
\hline 1 & 1 & -0.9 & 0.8 & -0.7 & 0.6 & -0.5 & 0.4 \\
\hline 2 & 1 & -0.900 & 0.800 & -0.700 & 0.600 & -0.500 & 0.400 \\
\hline 3 & & -0.900 & 0.806 & -0.692 & 0.598 & -0.500 & 0.402 \\
\hline
\end{tabular}

\begin{tabular}{ccccccc}
\hline$\gamma[g] \backslash g$ & 7 & 8 & 9 & 10 & 11 & 12 \\
\hline 1 & -0.3 & 0.2 & -0.1 & 0 & 0 & 0 \\
\hline 2 & -0.300 & 0.200 & -0.100 & 0.000 & 0.000 & 0.000 \\
\hline 3 & -0.302 & 0.197 & -0.107 & -0.003 & -0.001 & -0.003 \\
\hline
\end{tabular}

\section{EXTENSION BY RANDOM THINNING}

\section{A. Statement of the Problem}

In order to simplify the presentation, we shall restrict the following discussion to the very simple, but nevertheless typical, problem of constructing a UP SBRS which is also MA(1) with a given CF. Let us be reminded that the CF of a MA(1) signal of UP is defined by only one parameter and takes the general form

$$
\gamma[g]=\delta[g]+\gamma\{\delta[g+1]+\delta[g-1]\}
$$

The parameter $\gamma$ however is not arbitrary but must satisfy the condition $|\gamma|<1 / 2$ which is necessary to ensure that $\gamma[g]$ is non-negative definite. It is easy to verify that if this condition is not satisfied the Fourier transform of $\gamma[g]$ takes negative values and cannot be a PSD. Before describing the thinning method we shall present the limitations of the other known solutions. 


\section{B. Perfect clipping Method}

This method was partially introduced in Section II where it was shown that it can in some cases be useless because it leads to a CF that is not positive definite, yielding a PSD taking negative values. This appears in particular when the Gaussian input $X_{k}$ is a MA(1) signal. In this case the clipped signal $Y_{k}$ is a UP SBRS and the relation between the CFs of $X$ and $Y$ is $\gamma_{Y}[g]=(2 / \pi) \arcsin \left\{\gamma_{X}[g]\right\}$. This implies that if $X$ is a MA(1) signal, $Y$ is also MA(1) and their covariance are given by (26) where $\gamma_{X}$ and $\gamma_{Y}$ are related by $\gamma_{Y}=(2 / \pi) \arcsin \left(\gamma_{X}\right)$. This yields $\gamma_{X}=\sin \left[(\pi / 2) \gamma_{Y}\right]$. But the values of $\gamma_{Y}$ satisfying $1 / 3<\gamma_{Y}<1 / 2$ imply $\gamma_{X}>1 / 2$ which means, as indicated just before, that the corresponding CF is not NND. This elementary example shows that this method cannot generate a SBRS with the CF of a MA(1) signal defined by $\gamma_{Y}$ if $1 / 3<\gamma_{Y}<1 / 2$. This is the principal limitation of this procedure.

\section{Random Filtering}

It is well-known that a MA(1) signal can be generated by filtering a white noise in a RIF filter the impulse response of which has only two non-zero coefficients $h_{0}$ and $h_{1}$. This greatly simplifies the structure of the random filtering defined by (12). This expression is also limited to two terms and becomes

$$
X_{k}=B_{k} W_{k}+\bar{B}_{k} W_{k-1}
$$

where $W_{k}$ is a white SBRS taking the values \pm 1 and $B_{k}$ or $\bar{B}_{k}$ have the same meaning as in (4). It is clear that $X_{k}$ is a SBRS taking the values \pm 1 and it results from our assumptions that its CF $\gamma_{X}[g]$ is zero if $|g|>1$. It is then a $\mathrm{MA}(1)$ signal of unit power and with $\gamma_{X}[1]=p q$. But the maximum value of $p q$ when $p \in[0,1]$ is $1 / 4$, which means that this procedure cannot generate symmetric binary MA(1) signals with $\gamma_{X}[1]>1 / 4$. This means that this procedure is worse than that of clipping of Gaussian MA(1) signals. Let us show that it is possible to overcome this inconvenience by using an appropriate method of thinning.

\section{Random Thinning}

Let us first introduce this procedure. For this consider the signal $X_{k}$ defined by

$$
X_{k}=B_{k} X_{k-1}+\bar{B}_{k} W_{k}
$$

where $B_{k}$ has the same definition as in (4) or in (27). It results from the ideas appearing in [2] that $X_{k}$ is a SBRS signal with unit power but is also $\operatorname{AR}(1)$. Its covariance function is $\gamma_{X}[g]=p^{|g|}$, where $p$ is the probability that $B_{k}=1$. To pass from this signal to another $Y_{k}$ which is quasi MA(1) we can use the procedure of random thinning. 
The covariance between $X_{1}$ and $X_{2}$ is $p$ while the covariance between $X_{1}$ and $X_{D}$ is $p^{D}$. If $D$ is sufficiently large we can regard these values as being uncorrelated. This leads us to suppress all the values of $X$ from $X_{3}$ to $X_{D-1}$ and to define $Y$ so that $Y_{1}=X_{1}, Y_{2}=X_{2}, Y_{3}=X_{D}$, and $Y_{4}=X_{D+1}$. The same procedure of thinning can be applied for the values of $X_{k}$ corresponding to $k$ posterior to $D+1$ which yields a signal $Y_{k}$. Its first fundamental property is that it is of course still a SBRS like $X_{k}$ from which it is constructed. Furthermore, the covariance between successive values are either 0 or $p$.

Since the covariance between successive values of $Y_{k}$ is either 0 or $p$ with the same probability, its value measured by time averaging is equal to $p / 2$ and is approximatively zero for delays greater than 1 . This is why we use the term of quasi MA(1) signal, and also note that the quality of the approximation improves with $D$.

It is clear on this model that obtaining a covariance between successive values of $Y_{k}$ equal to $1 / 2$, which is the maximum possible for an $\mathrm{MA}(1)$ signal, would require that $p=1$. This is impossible because all the RVs $B_{k}$ would be equal to 1 and the model (28) would introduce a constant signal, which is without interest.

It is however interesting to verify these properties in a computer experiment. It is easy to generate trajectories of the signal $X_{k}$ given by (28). The results are summarized in Table 2 .

We realized by computer simulations various trajectories of the signals $X_{k}$ and $Y_{k}$ by applying the algorithms coming from their definition. For $X_{k}$ it is sufficient to apply (28) and the only arbitrary parameter of this algorithm is the probability $p$. For $Y_{k}$ it is also necessary to specify the value of the delay $D$ used for its definition. This is done by calculating the value $p^{D}$ which is the covariance between the values of $X$ for $k$ and $k+D$ in such a way that this covariance is smaller than the statistical error appearing in covariance measurements. For the three values of $p$ chosen in our experiments $(0.6,0.8$, and 0.9 ) the associated values of $D$ are such that $p^{D}$ are equal to $2 \times 10^{-7}, 1.5 \times 10^{-6}$, and $2.5 \times 10^{-5}$. We have thus verified that the resulting signals $X_{k}$ and $Y_{k}$ take only the values \pm 1 with a zero mean value, which means that they are effectively SBRS. The line "theory" indicates the value of the covariance given by $p^{g}$ for $g=0,1$, and 2 . The line $X_{k}$ displays the first three values of its covariance and it is the same presentation for the line $Y_{k}$. It appears in almost perfect agreement between theoretical values and measurements in the AR case or for $X_{k}$. For the line $Y_{k}$ we observe that the expected results are obtained within the statistical precision of our measurements. The values of $\gamma_{g}$ for $g=1$ are effectively the half of those obtained for $X_{k}$, while they are almost zero for $g>1$. We have verified that in this case the values of the CF of $Y_{k}$ are of the order of $10^{-6}$. It is clear that the precision of the measurements depends of the number of values of $X_{k}$ generated by (28) which is here of the order of $10^{7}$. A greater number of such values would yield a better precision. 
The main conclusion of these measurement is that the proposed method yields the possibility to generate SBRS of unit power that are $\mathrm{MA}(1)$ and for which the only nonzero value to the covariance function can take any value in the interval $[0,1 / 2[$.

\section{CONCLUSION}

The purpose of this paper was to introduce appropriate methods to generate binary symmetric random signals with given spectral properties. After justifying the interest of binary signals due to their use either in various problems of Information Theory or in number of digital communication or Signal Processing systems, we have noticed that the standard methods already known for generating random signals with given covariance properties and given marginal distribution are in general inefficient in the case of binary signals because they can lead to covariance functions that are not non-negative, which implies that power spectral densities (PSD or spectrum) take negative values. Since binary signals taking only the values \pm 1 are signals of unit power, we have first analyzed more precisely the structure of their PSD. In particular, in order to define the concept of spectral properties of a signal, we have introduced an equivalence relation between PSDs of signals with unit power leading to a partition of the space of PSDs in classes of equivalence. This was the basis of the definition of spectral properties meaning that two signals have the same spectral properties if their corresponding PSDs belong to the same class of equivalence. This partition is not only a theoretical mean but we have also shown that it can be interpreted in terms of signal processing by introducing a procedure allowing the generation of all the signals belonging to the equivalence class defined by a given spectrum. For this purpose we have introduced a new kind of signal filtering extending the classical concept of linear filter by using random impulse response. This procedure of statistical filtering can also be realized by computer simulation and used in a computer experiment yielding results confirming the validity of the method. This method was finally extended by using a procedure of random thinning sometimes used in simulations of point processes and computer experiments with MA(1) signals indicate very good performance of this method.

\section{Appendix : List of acronyms used in the text}

ARMA : Autoregressive and moving average,

$\mathrm{CF}$ : Covariance function,

CT : Continuous time,

DT : Discrete time,

IID : Independent and identically distributed,

MA(1) : Moving average of order 1, 
NND : Non-negative definite,

PDF : Probability density function,

PSD : Power spectral density,

RS : Random signal,

RV ; Random variable ;

SBRS : Symmetric binary random signal,

UP : Unit power. 
TABle 2. Covariance Functions of AR(1) And MA(1) RAndom Binary Signals

\begin{tabular}{|c|c|c|c|c|}
\hline & & $\gamma_{0}$ & $\gamma_{1}$ & $\gamma_{2}$ \\
\hline \multicolumn{5}{|l|}{$p=0.6$} \\
\hline & TheoryAR & 1 & 0.6 & 0.36 \\
\hline & $X_{k}$ & 1.0000 & 0.6003 & 0.3600 \\
\hline & $Y_{k}$ & 1.0000 & 0.3003 & 0.0013 \\
\hline \multicolumn{5}{|l|}{$p=0.8$} \\
\hline & TheoryAR & 1 & 0.8 & 0.64 \\
\hline & $X_{k}$ & 1.0000 & 0.8001 & 0.6403 \\
\hline & $Y_{k}$ & 1.0000 & 0.4016 & 0.0047 \\
\hline \multicolumn{5}{|l|}{$p=0.9$} \\
\hline & TheoryAR & 1 & 0.9 & 0.81 \\
\hline & $X_{k}$ & 1.0000 & 0.9000 & 0.8100 \\
\hline & $Y_{k}$ & 1.0000 & 0.4523 & $510^{-5}$ \\
\hline
\end{tabular}

\section{REFERENCES}

[1] Picinbono B., Random Signals and Systems, Englewood Cliffs, N.J. : Prentice Hall, 1993.

[2] Picinbono B., "ARMA signals with specified symmetric marginal Probability distribution,” IEEE Trans. Signal Processing, vol. 55-3, pp. 1542-1252, March 2010.

[3] Liu B., Munson D., "Generation of a random sequence having a jointly specified marginal distribution and autocovariance," IEEE Trans. on Acoustics, Speech and Signal Processing, vol. ASSP 30, pp. 973-983, December 1982.

[4] Sondhi M.M., "Random processes with specified spectral density and first-order probability density," Bell System Technical Journal, vol. 62, pp. 679-702, 1983.

[5] Conte E., Lops M., Ricci G., "Generation of random sequences with prescibed marginal distribution and autoCF," International Symposium on Communication Theory and its Applications, Warwick (Scotland), September 1991.

[6] Kay S., "Representation and generation of non-Gaussian wide-sense stationary random processes with arbitrary PSDs and a class of PDFs," IEEE Trans. Signal Processing, vol. 58-7, pp. 3448-3458, July 2010.

[7] Oman S. D. and Zucker D. M., "Modelling and generating correlated binary variables," Biometrika, vol. 88-1, pp. 287290, 2001.

[8] Qaqish B. F., "A family of multivariate binary distributions for simulating correlated binary variables with specified marginal means and correlations," Biometrika, vol. 90-2, pp. 455463, 2003.

[9] Bello, P., "Characterization of randomly time-variant linear channels," IEEE Trans. Comm. Syst., CS 11, p. 360-393, 1963.

[10] Tsatsanis, M., Giannakis, G., Zhou, G. "Estimation and equalization of fading channels with random coefficients," Signal Processing, 53, p. 211-229, 1996.

[11] Cambanis S., "Random filters which preserve the stability of random inputs," Advances in applied probability, 20, pp. 274-294, 1998. 

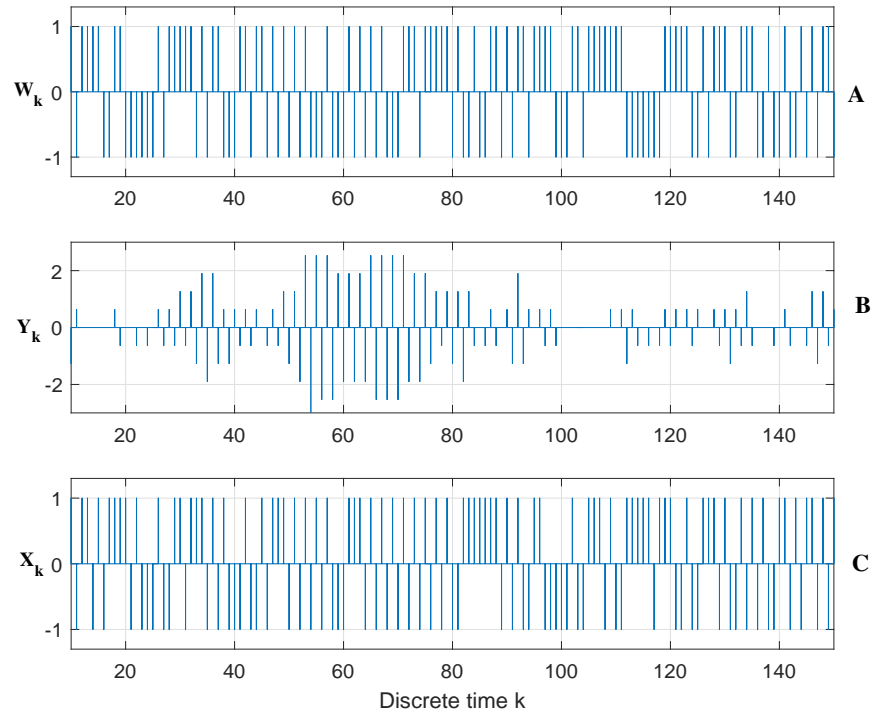

Fig. 1. Trajectories of simulated signals. 